\title{
Dystonic-Parkinsonian syndrome after cyanide poisoning: clinical and MRI findings
}

\author{
FRANCESCO CARELLA, MARIA PIA GRASSI, MARIO SAVOIARDO,* \\ PATRIZIA CONTRI, BARBARA RAPUZZI, ALFONSO MANGONI
}

\author{
From the Clinica Neurologica Ospedale L. Sacco and Istituto Neurologico C. Besta, ${ }^{*}$ Milan, Italy
}

SUMMARY Progressive Parkinsonism, dystonia and apraxia of eye opening were seen after cyanide poisoning. CT scan and MRI showed lesions in the basal ganglia, cerebellum and cerebral cortex consistent with reported pathological findings.

Parkinsonian signs and abnormal postures after cyanide poisoning have been described. ${ }^{12}$ Since cyanide poisoning is almost always fatal, reports of patients surviving to develop neurological signs are few. We report here the clinical and radiological features of one patient who developed Parkinsonism, dystonia and a disorder of eye opening following accidental cyanide poisoning.

\section{Case report}

A 46 year old woman and her husband were brought to the hospital after drinking from a bottle of a soft drink which had been adulterated with cyanide. The man died before arriving at the hospital. On admission, his wife was found to be in light coma, readily reacting to pain and moving her limbs without obvious asymmetry. Babinski's sign was present on the right. An analysis of gastric aspirates from the patient and her husband confirmed the presence of cyanide. CT scan and cerebrospinal fluid examination were normal. EEG showed marked diffuse slow activity, but was normal on discharge. The patient showed occasional decerebrate posturing, but slowly recovered in 2 weeks. She was transferred to a rehabilitation unit where she was found to have dysphonia and dysarthria, slight plastic limb hypertonia, slight right hemiparesis with right Babinski's sign and diffuse hyperactivity of tendon reflexes.

One year later, the patient was admitted to another hospital because of increasing speech and swallowing difficulties, drooling and unsteadiness of gait. CT scan showed only moderate cortical atrophy; EEG showed minor

Address for reprint requests: Francesco Carella, MD Clinica Neurologica Ospedale L. Sacco via G.B. Grassi, 74-20157 Milan, Italy

Received 29 January 1988 and in revised form 21 April 1988 Accepted 4 May 1988 non-specific changes in temporal leads. Neuropsychological examination showed an impairment of spatial and visual memory, poor visuo-perceptual performance and abstract reasoning. Subsequently, drooling and dysphagia became more marked and sustained abnormal postures of the mouth and tongue developed. At the age of 51, 5 years after poisoning, the patient was readmitted to our hospital. On examination, she showed a dystonic posture of mouth and tongue which was deviated to the right and twisted (fig. 1). Drooling was marked and swallowing was difficult; she was anarthric and markedly dysphonic. There was limitation of vertical gaze that improved with oculocephalic testing; there

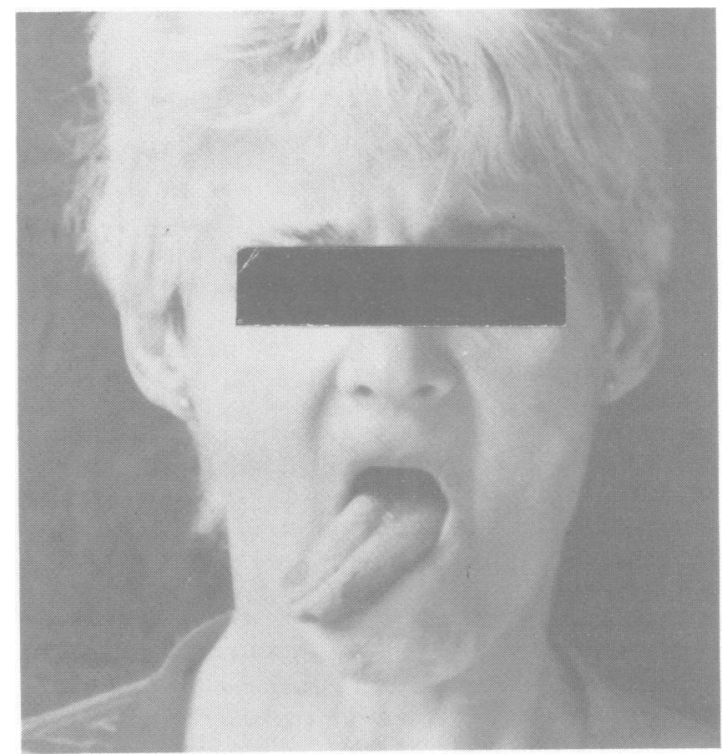

Fig 1 Dystonic posture of patient's tongue. 

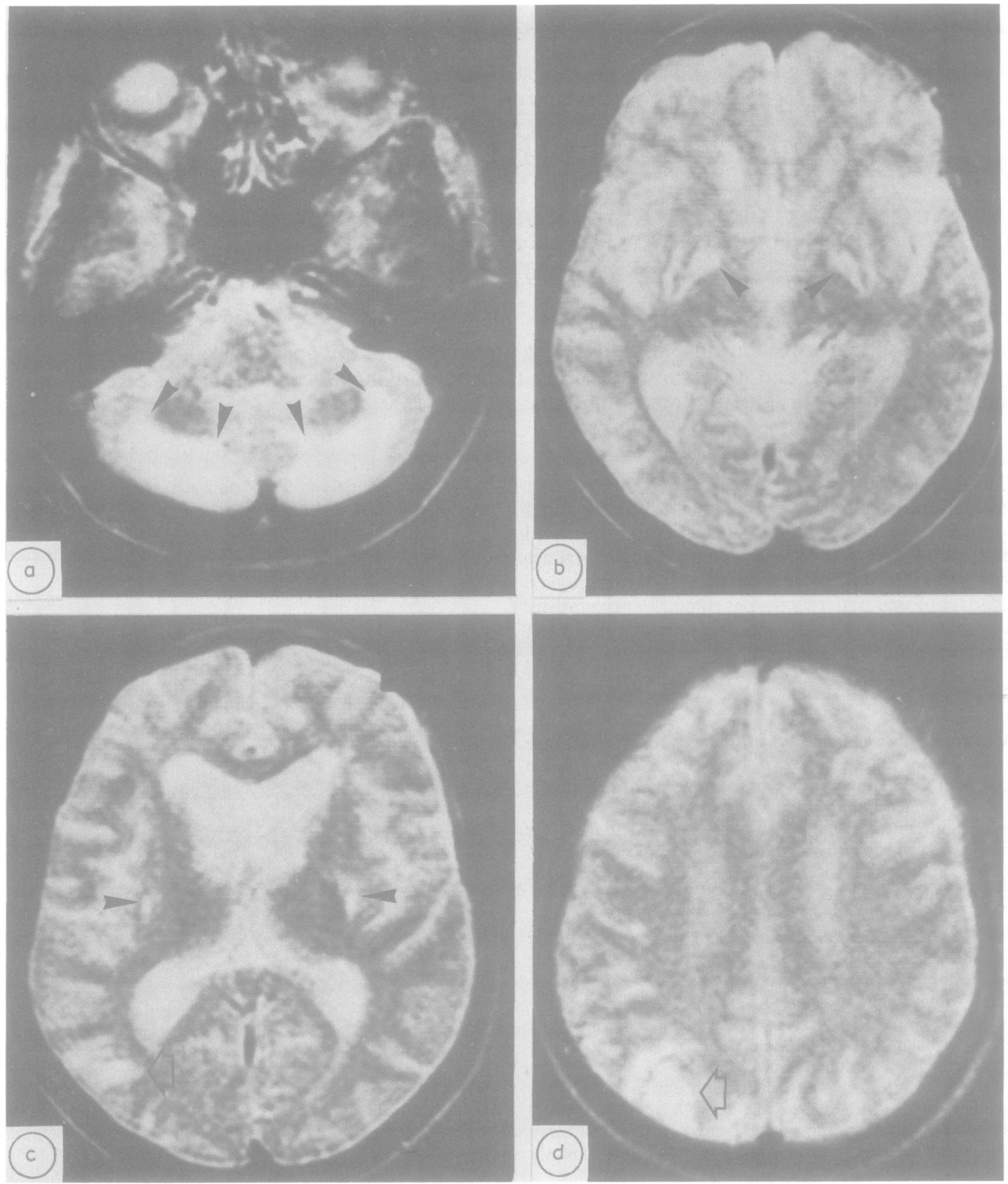

Fig 2 MRI Spin-Echo technique: TR $1800 \mathrm{~ms}$, TE $100 \mathrm{~ms}$. (a) high signal intensity in posterior cerebellar cortex and part of subcortical white matter may correspond to distal territory of posterior inferior cerebellar arteries and watershed areas between these vessels and superior cerebellar arteries (arrow heads). (b) and (c) high signal intensity in pallida and putamina (arrow heads). (c) and (d) abnormal signal in right cortical temporo-occipital and parietal watershed areas (arrows). Enlarged lateral ventricles. No central white matter demyelination. 
was also failure to maintain gaze to the left. On voluntary eye closure, the patient was frequently unable to open them. No orbicularis oculi contraction was evident. Eventually, the patient resorted to lifting her lids with her hands. Spontaneous blinking was decreased but otherwise normal. Moderate bradykinesia and slight plastic hypertonia were present, more marked on the right side. A slight right hemiparesis and Babinski's sign were present. Finger to nose testing was impaired on the left side. Standing and walking were wide-based and unsteady.

\section{Results}

All serum laboratory investigations, including copper studies, hexosaminidase and beta-galactosidase activity, were normal. Cerebrospinal fluid examination was normal including IgG index and isoelectrofocusing. EEG showed diffuse irritative activity most prominent in the left temporo-occipital leads. Visual evoked responses showed normal latency but decreased amplitude of P 100 . Brainstem auditory evoked responses (BAER) showed delay to the peak of VI wave. Psychometric testing showed no evidence of further deterioration of the patient's performance when compared with previous tests. There was continued evidence of impaired visual and constructional ability as well as abstract reasoning. CT scan showed diffuse atrophy most marked in the posterior fossa; all the ventricles were dilated. Two hypodense areas in the basal ganglia, primarily involving the pallida, were present. There were also low density areas in the posterior portions of the cerebellar hemispheres and in the right parietal cortex. MRI performed with a 0.35 tesla magnet confirmed atrophy of the cerebellum and the cerebral hemispheres with marked ventricular enlargement. Abnormal signal, hyperintense in $T_{2}$ weighted images, was present in both cerebellar hemispheres, involving the posterior cortical areas with extension to the subcortical white matter. Similar abnormal hyperintense signal was present in the cerebral hemispheres, the pallida, the posterior parts of the putamina and the right parietal cortex (fig. 2).

The patient was discharged on trihexyphenidyl $6 \mathrm{mg} /$ day with improvement in bradykinesia and the eyelid opening apraxia; she was frequently able to open her eyes voluntarily provided she had not closed them very tightly. Trial withdrawal of trihexyphenidyl worsened both these complaints. Levodopa was not tolerated by the patient who experienced confusion and spontaneously stopped the drug before being re-examined. She was restarted on trihexyphenidyl up to $8 \mathrm{mg} /$ day with improvement; higher doses were not tolerated as they caused constipation.

\section{Discussion}

Our patient showed Parkinsonian features, an abnormal posture of the mouth and tongue and a disorder of eye opening. Both Parkinsonism ${ }^{1}$ and abnormal postures ${ }^{2}$ have been described after cyanide poisoning and are consistent with the pathological and radiological evidence of striaial damage. Progression of dystonia after a static insult is well known and may possibly be due to aberrant sprouting of surviving neurons. ${ }^{3}$ An impairment of lid opening described as apraxia of lid opening has also been described after cyanide poisoning. ${ }^{4}$ The anatomical basis of apraxia of lid opening is not well understood; its physiopathologic mechanisms may differ under various circumstances; it has been described in a variety of conditions such as Huntington's disease, Parkinson's disease, progressive supranuclear palsy, the Shy-Drager syndrome, atypical Parkinsonism, neuroacanthocytosis and hemispheric damage. ${ }^{4-9}$ Involuntary levator palpebrae inhibition may account for apraxia of eyelid opening in patients with extrapyramidal disease. $^{7}$

In our patient there was clinical and radiological evidence of striatal involvement. In addition, clinical and neurophysiological evidence suggested midbrain damage: the impairment of eye opening was associated with limitation of upward gaze and with delay of VI wave of BAER. Interestingly, the pars reticulata of the substantia nigra, which is known to participate in the control of eye movements in monkeys, ${ }^{10}$ has been found to be damaged after cyanide poisoning. ${ }^{1}$ Our observation suggests that eyelid opening apraxia is a non-specific phenomenon which may be seen in combined striatal and midbrain damage. In agreement with the pathological report by Uitti et al, and with the CT findings observed by Finelli, our CT and MRI findings showed evidence of brain atrophy and lenticular lesions. ${ }^{12}$ There was also evidence suggestive of distal and watershed infarcts in the cerebellum and parietal cortex which were better demonstrated by MRI. No central demyelination was seen; in this respect our radiological findings are in agreement with the pathological observation by Utti et al. ${ }^{1}$

Anticholinergic treatment improved apraxia of eye opening in our patient, suggesting that cholinergic pathways may be involved in some patients. Improvement after amantadine and levodopa was reported in the case discussed by Picard and Wray who suggested that the impairment of lid opening was due to retarded initiation of lid movement. ${ }^{6}$ Brusa $e t$ $a l$, however, reported persistence of apraxia of eyelid opening despite pharmacological treatment in patients with idiopathic Parkinson's disease. ${ }^{5}$ In our patient the apraxia of eye opening improved together with global bradykinesia.

In conclusion, our case confirms that Parkinsonism, dystonia and lid opening apraxia may evolve after cyanide poisoning. MRI in this condition may be the 
most sensitive investigation for demonstrating the location and extent of the lesions induced by cyanide.

We thank Dr G Scialfa who performed the MRI study at the Casa di Cura S Pio X, Milan.

\section{References}

1 Uitti RJ, Rajput AH, Ashenhurst EM, Rozdilsky B. Cyanide-induced Parkinsonism: a clinicopathologic report. Neurology 1985;35:921-5.

2 Finelli PF. Changes in the basal ganglia following cyanide poisoning. J Comput Assist Tomogr 1981;5:755-6.

3 Burke RE, Fahn S, Gold AP. Delayed-onset dystonia in patients with "static" encephalopathy. J Neurol Neurosurg Psychiatry 1980;43:789-97.

4 Goldstein JE, Cogan DG. Apraxia of lid opening. Arch Ophthalmol 1965;73:155-9.
5 Brusa A, Mancardi G, Meneghini S, Picardo A, Brusa G. Apraxia of eye opening in idiopathic Parkinson's disease. Neurology 1986;36:134.

6 Case records of the Massachusetts General Hospital: Case 32-1975. N Engl J Med 1975;293:346-52.

7 Lepore FE, Duvoisin RC. "Apraxia" of eyelid opening: an involuntary levator inhibition. Neurology 1985; 35:423-7.

8 Bonaventura I, Matias-Guiu J, Cervera C, Codina Puiggros A. Neuroacanthocytosis syndrome, apraxia of eyelid opening, and progressive supranuclear palsy. Neurology 1986;36:1276.

9 De Renzi E, Gentilini M, Bazolli C. Eyelid movement disorders and motor impersistence in acute hemisphere disease. Neurology 1986;36:414-8.

10 Hikosaka $\mathrm{O}$, Wurtz RH. Visual and oculomotor functions of monkey substantia nigra pars reticulata. IV. Relation of substantia nigra to superior colliculus. $J$ Neurophysiol 1983;49:1285-1301. 